\title{
Effects of repeated gestation and lactation on milk $n-6$ fatty acid composition in rats fed on a diet rich in 18:2n-6 or 18:3n-6
}

\author{
BY YUNG-SHENG HUANG, PETER R. REDDEN, DAVID F. HORROBIN, \\ SANDRA CHURCHILL AND BARBARA PARKER \\ Efamol Research Institute, Kentville, Nova Scotia B4N 4H8, Canada \\ AND RON P. WARD AND DAVID E. MILLS \\ Department of Health Studies, University of Waterloo, Waterloo, Ontario N2L 3G1, Canada
}

(Received 26 March 1991-Accepted I August 1991)

\begin{abstract}
The present study examined the effect of repeated gestation and lactation on the levels of long-chain n-6 polyunsaturated fatty acids in rat milk fat, and examined whether such levels might be modulated by supplementing the diet of the lactating dams with either $(\mathrm{g} / \mathrm{kg}) 50$ safflower oil (SFO; containing $800 \mathrm{~g}$ $18: 2 n-6 / \mathrm{kg}$ ), or 50 evening primrose oil (EPO; containing $720 \mathrm{~g} 18: 2 n-6$ and $90 \mathrm{~g} 18: 3 n-6 / \mathrm{kg}$ ). The milk was collected at three different times (days 1,8 and 15) in each given lactation period from female Sprague-Dawley rats which were successively bred for four pregnancies and lactations. Results showed that dietary fat and breeding frequency had no significant effects on milk triacylglycerol content, but they modified the pattern of milk fatty acids in both triacylglycerol and phospholipid fractions. After three or four successive breedings rats fed on EPO produced milk containing less saturated but more monounsaturated and polyunsaturated fatty acids compared with those fed on SFO. During the course of lactation the levels of $n-6$ metabolites, e.g. 18:3n-6, 20:3n-6 and 20:4n-6, in milk fat declined progressively. However, they were consistently higher in the EPO group than in the SFO group. These findings suggest that the levels of long-chain $n-6$ metabolites in the milk fat may be increased through supplementing the maternal diet with $18: 3 n-6$.
\end{abstract}

Maternal diet: Milk triacylglycerols: $n-6$ Polyunsaturated fatty acids

Long-chain polyunsaturated fatty acids (PUFA) are required for the normal post-natal development of the newborn (Clandinin et al. 1980). After birth the PUFA, particularly the $n-6$ and $n-3$ essential fatty acids (EFA) which cannot be synthesized de novo by the animals, are supplied to the neonate through milk. In milk the EFA content changes during the course of lactation, varies between species and strains, and may be affected by the fat in the maternal diet (Jenness, 1974; Jensen et al. 1978; Brandorff, 1980; Grigor \& Warren, 1980; Jensen, 1989; Mills et al. 1990).

Studies in hurnans have shown that frequent reproduction with short recuperative periods does not significantly affect the milk fat concentration, but it reduces the maternal fat stores (Prentice et al. 1981; Merchant \& Martorell, 1988). The explanation for this change is that the mother buffers the nutrient requirement of the infant by depleting her own fat reserve to support fetal growth. In a preliminary study we compared the liver lipid fatty acid composition of four-time successive bred dams $(n 8)$ and age-matched virgin female rats ( $n 8)$ maintained on a semi-purified diet (Teklad Test Diets, Madison, Wisconsin) supplemented with $50 \mathrm{~g}$ safflower oil $/ \mathrm{kg}$ (SFO; containing $800 \mathrm{~g} 18: 2 n-6 / \mathrm{kg}$ ). Despite an ample supply of $18: 2 n-6$ in the diet, a significant reduction of $18: 2 n-6$ in liver triacylglycerols (TG) and of 20:4n-6 in plasma TG and phospholipids (PL) and in liver PL 


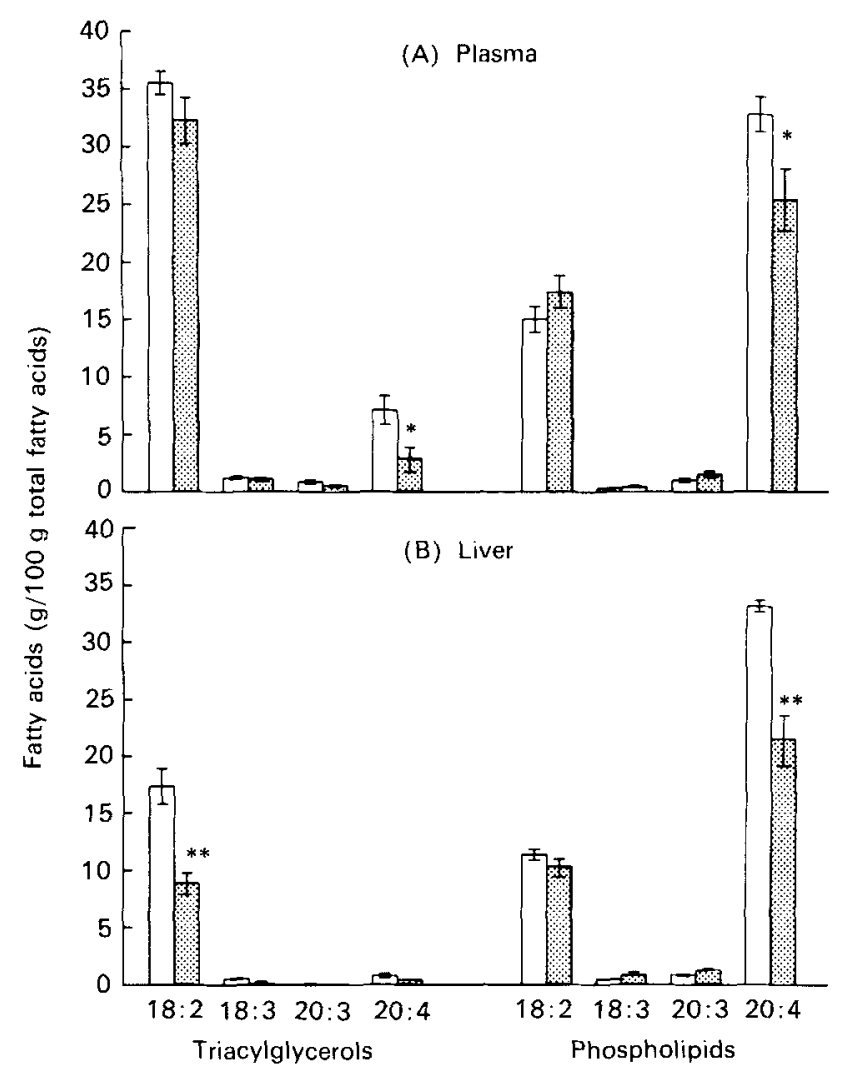

Fig. 1. Composition (g/100 g total fatty acids) of $18: 2 n-6,18: 3 n-6,20: 3 n-6$ and $20: 4 n-6$ in (A) plasma and (B) liver triacylglycerols and phospholipids of four-times-bred dams ( $\mathrm{O}$ ) in comparison with the age-matched virgin females ( $\square$ ). All animals were fed on a semi-purified diet supplemented with $50 \mathrm{~g}$ safflower oil $/ \mathrm{kg}$. Results are means with their standard errors, represented by vertical bars, for eight animals. Mean values for four-times-bred dams were significantly different from those for virgin females: ${ }^{*} P<0 \cdot 05,{ }^{* *} P<0.01$. For details of diets see Table 1 .

was observed (Fig. 1). A decrease in 20:4n-6 might be due either to maternal depletion of this fatty acid, or to insufficient conversion of $18: 2 n-6$ to $20: 4 n-6$. It is of interest, therefore, to examine whether such changes may lead to a reduced long-chain $n-6$ PUFA content in milk.

In the present study we examined the effects of successive breeding on the composition of milk long-chain PUFA, and studied whether such effects could be modified by changing the maternal diet. The dams were bred for four consecutive pregnancies and lactation periods while being given a regular chow diet supplemented with $50 \mathrm{~g}$ SFO or evening primrose oil (EPO; containing $720 \mathrm{~g} 18: 2 n-6$ and $90 \mathrm{~g} 18: 3 n-6 / \mathrm{kg}$ ) $/ \mathrm{kg}$. Since $18: 3 n-6$ bypasses the rate-limiting $\Delta^{6}$-desaturation step in EFA metabolism, supplementation with EPO, which contains $18: 3 n-6$, might facilitate the synthesis of long-chain $n-6$ metabolites in the dams. Previously Hassam \& Crawford (1976) increased the $n-6$ fatty acid levels in rat milk by feeding $18: 3 n-6$ rich oil to the dams. In the present study the levels of $n-6$ fatty acids in milk sampled at different lactation times (days 1,8 and 15), corresponding to initial colostral secretion, mid-lactation and late-lactation, were analysed. Although semisynthetic and purified diets which contain defined nutrient compositions were frequently used in fat-supplementation studies, we elected to use the regular chow and not the semi- 
Table 1. Fatty acid composition ( $\mathrm{g} / 100 \mathrm{~g}$ total fatty acids) of ground rodent laboratory chow* supplemented with $50 \mathrm{~g}$ safflower or evening primrose oil/kg

\begin{tabular}{llc}
\hline Oil supplement ... & Saffower & Evening primrose \\
\hline $14: 0$ & $0 \cdot 9$ & $1 \cdot 0$ \\
$16: 0$ & $13 \cdot 1$ & $15 \cdot 0$ \\
$16: 1 n-7$ & $1 \cdot 2$ & $1 \cdot 4$ \\
$18: 0$ & $4 \cdot 9$ & $4 \cdot 6$ \\
$18: 1 n-9$ & $17 \cdot 4$ & $15 \cdot 4$ \\
$18: 1 n-7$ & $3 \cdot 1$ & $3 \cdot 6$ \\
$18: 2 n-6$ & $58 \cdot 0$ & $53 \cdot 0$ \\
$18: 3 n-6$ & - & $5 \cdot 0$ \\
$18: 3 n-3$ & $1 \cdot 1$ & $0 \cdot 8$ \\
Others & $0 \cdot 3$ & $0 \cdot 2$ \\
\hline
\end{tabular}

* The constituents in laboratory chow (5001; Ralston Purina) as shown by the supplier ( $\mathrm{g} / \mathrm{kg}$ ): protein 230 , fat 45 , ash 80 and mineral 25 .

$\dagger$ Include trace amounts of $20: 1,20: 5 n-3$ and $22: 6 n-3$.

synthetic diet. First, semi-purified diet contains a large proportion of sucrose $(700 \mathrm{~g} / \mathrm{kg})$ which is known to increase the synthesis and incorporation of short- and medium-chain fatty acids (Linzell et al. 1969; Spincer et al. 1969) and might mask any changes in the longchain $n$-6 PUFA content of the milk. Second, a normal rat diet was chosen for these experiments in preference to a semi-synthetic diet as it was thought that the results might then be more readily applicable to human beings.

\section{MATERIALS AND METHODS}

\section{Animals}

Weanling male and female Sprague-Dawley rats (3 weeks old; Charles River Breeding Laboratories, Montreal, Quebec) were fed ad lib. a ground rodent laboratory chow (5001; Ralston Purina Co., St Louis, Missouri, USA) supplemented with SFO ( $50 \mathrm{~g} / \mathrm{kg})$, and maintained on a $12 \mathrm{~h}$ light-dark cycle. Breeding began when the animals reached 12 weeks of age. Female rats $(n 40)$ were housed together with males during one sexual cycle. Conception was confirmed by the presence of a vaginal plug. The pregnant females were then separated from the males during the gestation period and caged individually. They were randomly divided into two groups $(n 20)$. The first group was maintained on the same SFO-supplemented diet while the second group was given a diet supplemented with $50 \mathrm{~g}$ EPO $/ \mathrm{kg}$. Once animals were assigned to one specific diet they received the same diet throughout the experiments. Table 1 shows that the fatty acid compositions of these two diets were very similar with the exception that the EPO diet contained $50 \mathrm{~g} 18: 3 n-6 / \mathrm{kg}$. The diets were prepared twice weekly and stored at $-20^{\circ}$. The gestation period was approximately 3 weeks, and the day of parturition was designated as day 1 of lactation. In each litter the pups were culled to eight. After 3 weeks of lactation the pups were weaned and the dams were bred immediately. The gestation-lactation cycle (approximately 6 weeks) for each dam was repeated four times. During each cycle milk samples were collected on days 1,8 and 15 .

\section{Milk collection}

For milk collection (09.00-11.00 hours) the dams were separated from the pups for at least $1 \mathrm{~h}$. After that they were anaesthetized lightly with diethyl ether and injected intraperitoneally with $0.5 \mathrm{IU}$ oxytocin. Milk samples $(0.5-1 \mathrm{ml})$ were collected using a 
gentle sucking apparatus as described by McBurney et al. (1964). In some instances the volumes of milk from dams which had already fed their pups before sampling were very low. As the parity number increased the conception rate decreased and, as a result, the number of dams available for milk sampling was also reduced. Thus, we selected only ten samples from each collection for the subsequent lipid analysis. As lactation in rats was limited to 3 weeks, the time (days 1, 8 and 15 post partum) selected for milk sampling was designed to represent the initial (or colostral) secretion, the mid-lactation milk, and the latelactation milk. Milk samples were stored at $-20^{\circ}$ under nitrogen.

\section{Lipid analysis}

The concentration of TG in the milk after dilution with 9 vol. saline $(9 \mathrm{~g}$ sodium chloride/l), was determined enzymically (Grossman et al. 1976) using a TG PAP kit (MAkit 30; Roche) and a centrifugal analyser (Cobas-Bio; Hoffmann-La Roche Ltd, Vaudreuil, Quebec). Before lipid analysis, milk samples were warmed to $37^{\circ}$ and mixed evenly. Total milk lipids were extracted using the method described by Folch et al. (1957). TG were separated by thin-layer chromatography (TLC) using the solvent system, hexane-diethyl ether-acetic acid $(80: 20: 1$, by vol.). No significant amounts of free fatty acids were observed, indicating that no apparent autolysis of milk fats occurred during the storage. Transesterification of the TG fraction was performed with boron trifluoride-methanol (Morrison \& Smith, 1964). Fatty acid methyl esters were extracted into hexane and analysed using a Hewlett-Packard gas-liquid chromatograph (model 5890; Mississauga, Ontario) equipped with a flame-ionization detector and a $50 \mathrm{~m}$ fused silica capillary column coated with $0.25 \mu \mathrm{m}$ Supelcowax 10 (Supelco, Bellefonte, Pennsylvania, USA) (Huang et al. 1991). The injector and detector temperature was $230^{\circ}$. Fatty acid methyl esters were identified by comparing their retention times with those of authentic standards (Nu-ChekPrep, Inc., Elysian, MN, USA and Supelco, Inc., Bellefonte, PA, USA). Results are expressed as $\mathrm{g} / 100 \mathrm{~g}$ total fatty acid.

\section{Statistics}

All results are expressed as the mean with their standard error for each group. Significant differences between dietary groups and over lactation time were determined by a two-way analysis of variance. Where the diet and lactation time interaction was significant $(P<$ $0 \cdot 05$ ), further comparison was made using the Newman-Keuls test. Statistical differences between the two dietary groups were also examined using the Student's $t$ test (Snedecor \& Cochran, 1967).

\section{RESULTS}

\section{Animals}

The conception rates in the first three matings were similar in the two dietary groups (SFO v. EPO): $100 \%$ for the first and second mating, and $95 \%$ for the third mating. The conception rate for the fourth mating was $90 \%$ in the EPO group and $79 \%$ in the SFO group. On all four occasions the litter sizes were similar in both dietary groups (ranging from 13.4 to 14.4 in the EPO group and from 13.0 to 13.8 in the SFO group). Furthermore, the daily fat intake (approximately $120 \mathrm{~g} / \mathrm{kg}$ body-weight) was not significantly different between the two dietary groups during the course of the study.

\section{Milk TG content}

Fig. 2 shows the TG concentrations in milk samples collected on days 1, 8, and 15 of each lactation from four successive lactation cycles. During each cycle (except for the fourth) the TG concentrations in day 8 milk samples were consistently increased, whereas those in day 


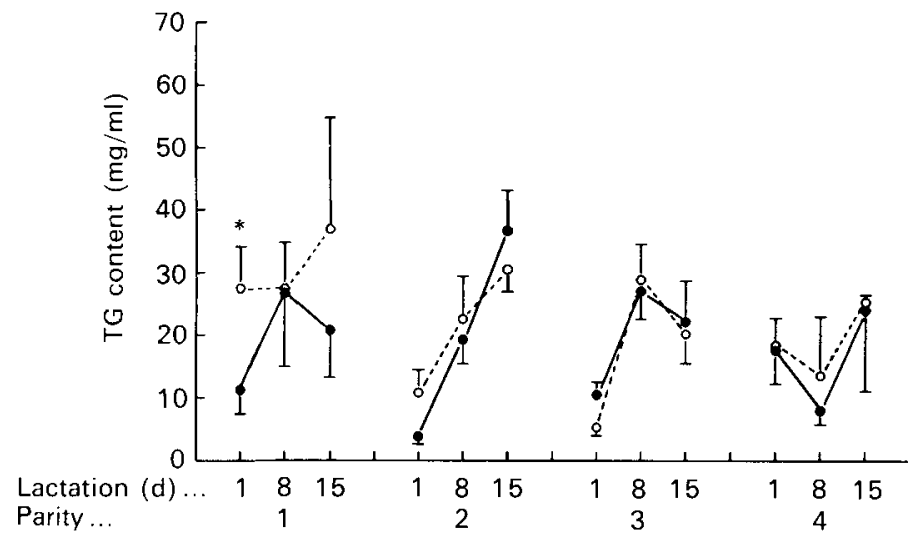

Fig. 2. Milk triacylglycerol (TG) concentrations $(\mathrm{mg} / \mathrm{ml})$ on days 1,8 and 15 of lactation collected from the dams (values are presented in the order of once-, twice-, three- and four-times-bred animals) fed on a diet supplemented with either safflower oil (SFO; O) or evening primrose oil (EPO; $)$ ). Results are means with their standard errors, represented by vertical bars, for ten animals. Mean values for SFO were significantly different from those for EPO: $* P<0.05$. For details of diets, see Table 1 .

15 samples were either increased or reduced. Dietary fat (SFO $v$. EPO) had no significant effects on milk TG content, except that of day 1 milk where levels were higher in the oncebred SFO-fed dams than in the once-bred EPO-fed dams.

\section{Fatty acid composition of milk $T G$}

The fatty acid composition $(\mathrm{g} / 100 \mathrm{~g})$ of milk TG is shown in Fig. 3. Saturated fatty acids (SFA) constituted approximately $35-45 \mathrm{~g} / 100 \mathrm{~g}$, monounsaturated fatty acids (MUFA) $15-20 \mathrm{~g} / 100 \mathrm{~g}$, and $n-3$ and $n-6$ PUFA $40-45 \mathrm{~g} / 100 \mathrm{~g}$ total fatty acids in milk TG. The levels of SFA (sum of 10:0, 12:0, 14:0,16:0 and 18:0) in milk TG were modulated by the type of maternal diet and the breeding frequency but less consistently by the day of lactation (Fig. 3(A)). The SFA levels (particularly 10:0 and 12:0) obtained from once- or twice-bred dams were lower in those animals fed on SFO than in those fed on the EPO diet, whereas those three- or four-times-bred dams had lower SFA levels when fed on EPO than when fed on the SFO diet.

The effects of dietary fats, days of lactation and the parity number on the levels of total MUFA in milk TG are shown in Fig. 3(B). In general, the levels of MUFA were not affected by the length of lactation, but they were affected by breeding frequency and the type of maternal diet. They were lower in milk obtained from the EPO-fed dams bred only once or twice than from those bred three or four times. The opposite results were found in milk samples obtained from the SFO-fed group. Between the two dietary groups, the levels of MUFA were lower in the EPO-fed than in the SFO-fed dams bred only once or twice, but they were not different in those bred three or four times.

The changes of $n-3$ and $n-6$ PUFA in milk TG in response to the effects of breeding frequency, day of lactation and maternal diet are shown in Fig. 3 (C and D) respectively. Although the levels of $n$-3 PUFA (ranging between 1.0 and $1.4 \mathrm{~g} / 100 \mathrm{~g}$ fatty acids) were significantly lower than those of $n-6$ PUFA (ranging between 37 and $45 \mathrm{~g} / 100 \mathrm{~g}$ total fatty acids), both $n-3$ and $n-6$ PUFA responded similarly to changes in dietary fat and breeding frequency. They remained more or less stable at different lactation times, but responded strikingly to different dietary fats and the parity number. In the once- and twice-bred dams 

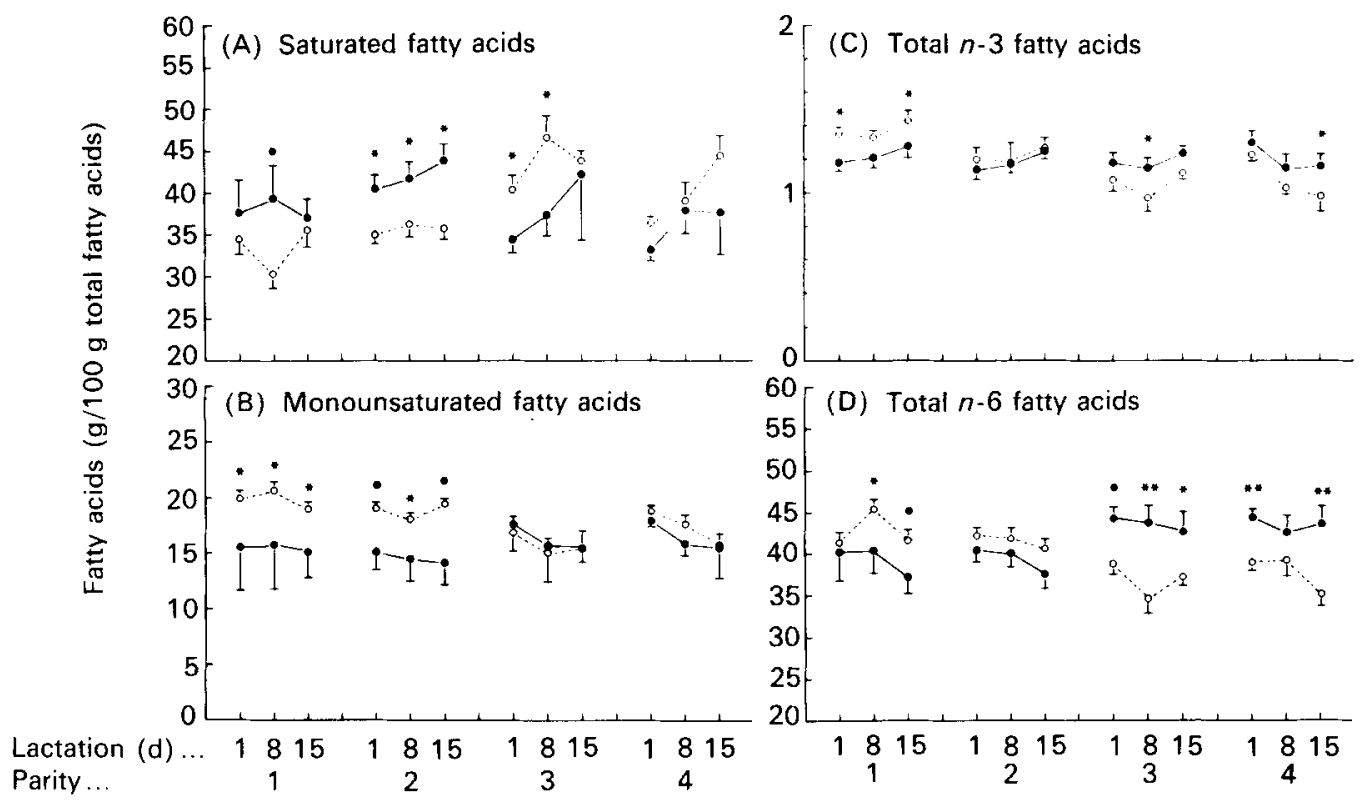

Fig. 3. Composition (g/100 g total fatty acids) of (A) saturated fatty acids, (B) monounsaturated fatty acids, (C) total $n-3$ and (D) total $n-6$ polyunsaturated fatty acids in rat milk triacylglycerol fraction on days 1,8 , and 15 of lactation. Milk samples were collected from the dams (values are presented in the order of once-, twice-, three- and four-times-bred animals) fed on a diet supplemented with either safflower oil (SFO; $O$ ) or evening primrose oil $(\mathrm{EPO} ; \mathrm{O})$. Results are means with their standard errors, represented by vertical bars, for ten animals. Mean values for SFO were significantly different from those for EPO: ${ }^{*} P<0 \cdot 05,{ }^{* *} P<0.01$. For details of diets, see Table 1.

the levels of $n-3$ and $n-6$ PUFA in milk TG were higher in the SFO group than in the EPO group, whereas in the three- and four-times-bred dams they were lower in the SFO group than in the EPO group.

The detailed changes of individual $n-6$ fatty acids in milk TG are shown in Fig. 4 . The levels of $18: 2 n-6$, which represents approximately $90 \%$ of total $n-6$ PUFA, correlated well with the changes shown by total $n-6$ PUFA (Fig. 4(A)). They were not affected by the lactation time, but they were significantly modulated by the maternal dietary fat and by the parity number of the dams. As the parity number increased the level of $18: 2 n-6$ increased in the EPO group whereas it fell in the SFO group.

Fig. 4(B-D) also shows that the levels of $18: 3 n-6,20: 3 n-6$ and $20: 4 n-6$ were, in general, consistent in day 1 milk sampled from each gestation-lactation cycle. As lactation continued (days 8 and 15) the levels of $n-6$ fatty acids fell, although $18: 3 n-6$ in milk TG obtained from the EPO-fed dams remained constant. The levels of all three fatty acids were consistently higher in milk collected from the EPO-fed dams than from the SFO-fed dams.

\section{Fatty acid composition of milk $P L$}

$\mathrm{PL}$, in comparison with $\mathrm{TG}$, constitute only a very small $(10 \mathrm{~g} / \mathrm{kg})$ but constant portion of milk fats (values not shown). The detailed changes of PL $n-6$ fatty acids in response to dietary fat and breeding frequency are summarized in Fig. 5. Generally, the levels of $18: 2 n$ 6 (Fig. 5(A)) and 18:3n-6 (Fig. 5(B)) increased, whereas those of 20:3n-6 (Fig. 5(C)) and 20:4n-6 (Fig. 5(D)) remained constant as the lactation proceeded. Similar to milk TG, the levels of 18:3n-6 (Fig. 5(B)), 20:3n-6 (Fig. 5(C)) and 20:4n-6 (Fig. 5(D)) were consistently higher in milk collected from the EPO-fed dams than in samples from the SFO-fed dams. 

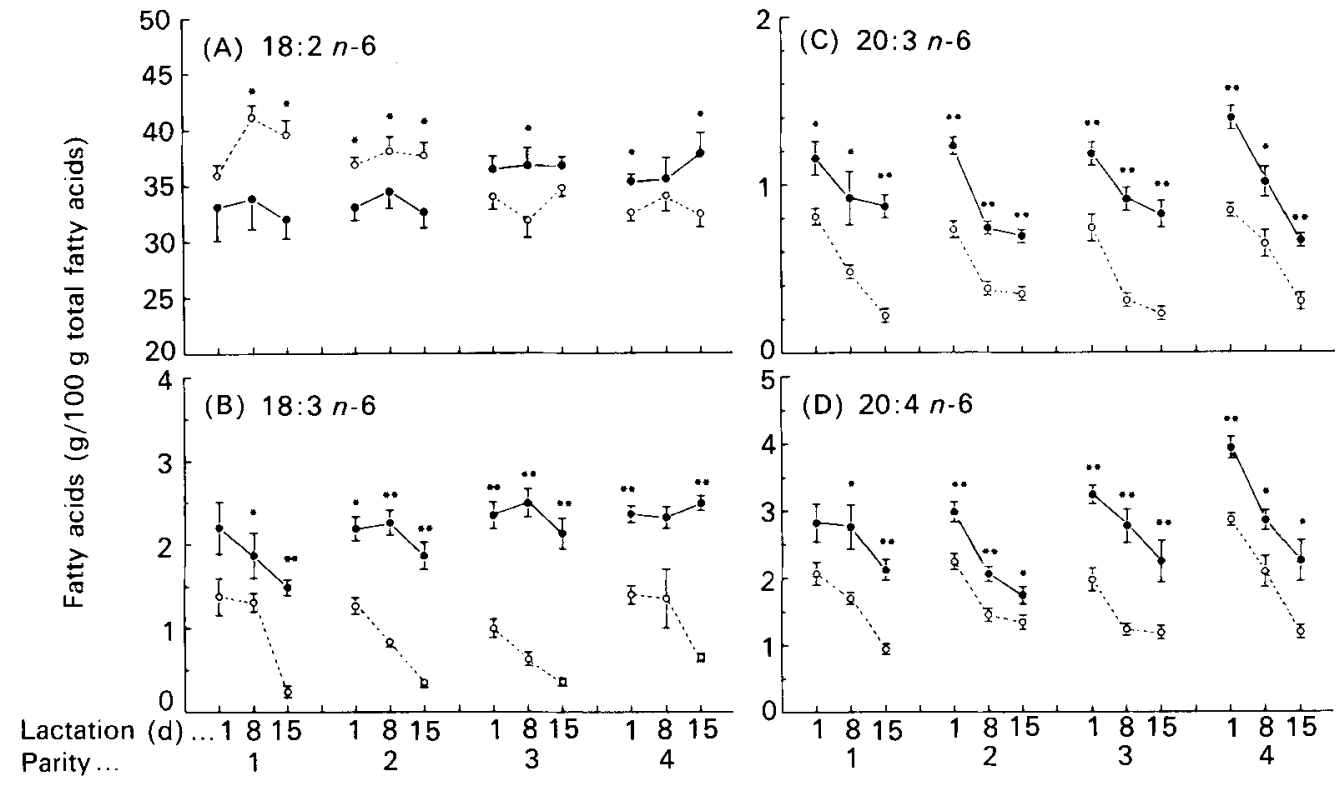

Fig. 4. Composition (g/100 g total fatty acids) of (A) $18: 2 n-6$, (B) $18: 3 n-6$, (C) $20: 3 n-6$ and (D) $20: 4 n-6$ in rat milk triacylglycerols on days 1,8 and 15 of lactation. Milk samples were collected from the dams (values are presented in the order of once-, twice-, three- and four-times-bred animals) fed on a diet supplemented with either safflower oil (SFO; $O$ ) or evening primrose oil (EPO; ). Results are means with their standard errors, represented by vertical bars, for ten animals. Mean values for SFO were significantly different from those for EPO: ${ }^{*} P<0.05,{ }^{* *} P<0.01$. For details of diets, see Table 1 .

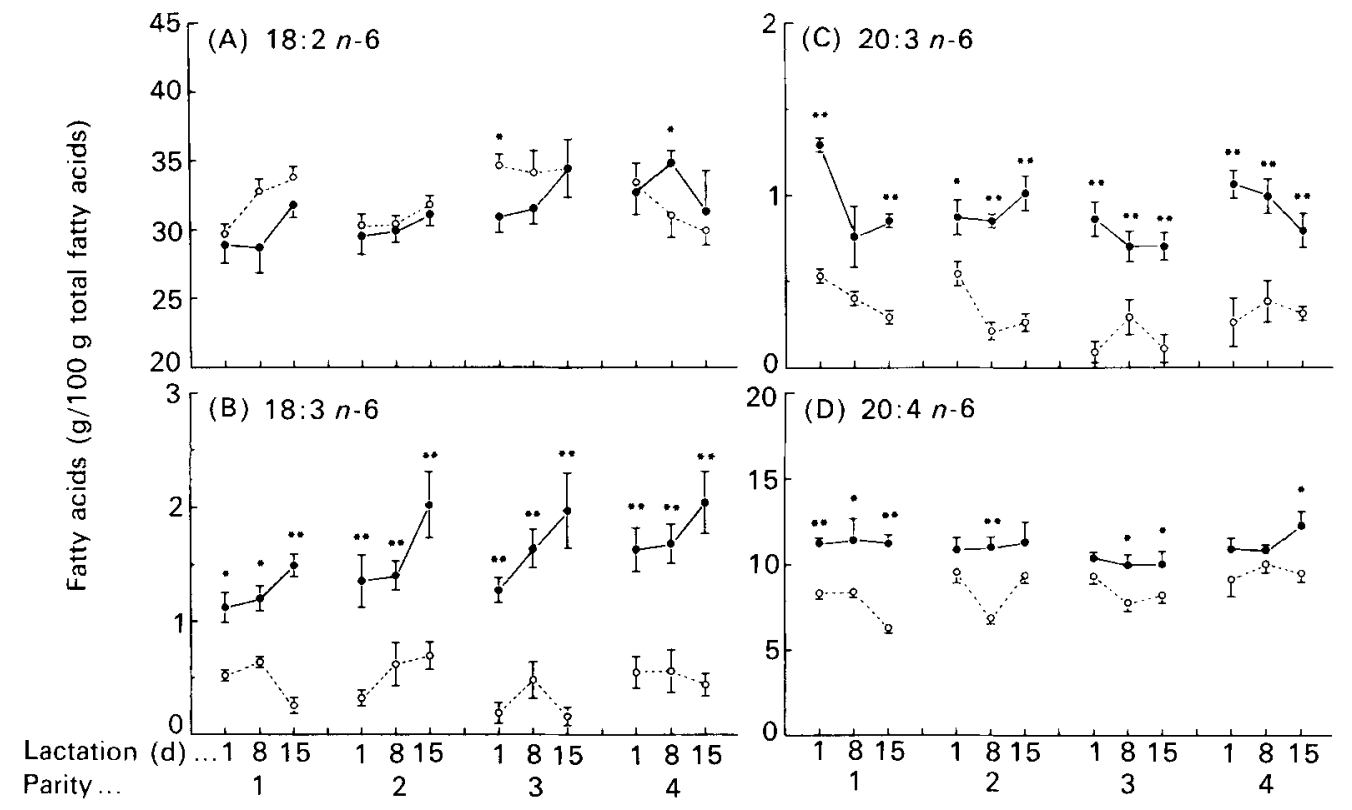

Fig. 5. Composition (g/100 g total fatty acids) of (A) $18: 2 n-6$, (B) $18: 3 n-6$, (C) $20: 3 n-6$ and (D) $20: 4 n-6$ in rat milk total phospholipids on days 1,8 and 15 of lactation. Milk samples were collected from the dams (values are presented in the order once-, twice-, three- and four-times bred animals) fed on a diet supplemented with either safflower oil $(\mathrm{SFO} ; O)$ or evening primrose oil $(\mathrm{EPO} ; \mathbf{O})$. Results are means with their standard errors for ten animals. Mean values for SFO were significantly different from those for $\mathrm{EPO}:{ }^{*} P<0.05,{ }^{* *} P<0 \cdot 01$. For details of diets, see Table 1. 


\section{DISCUSSION}

The first objective of the present study was to examine whether successive breeding and lactation affected the fat content and the fatty acid composition of rat milk. Since TG constitutes the major portion ( $>980 \mathrm{~g} / \mathrm{kg}$ ), whereas PL represents only a small but rather constant proportion of milk lipids (Morrison, 1968; Harzer et al. 1983), the changes in milk lipids reflected mainly the changes in milk TG. In the present study the milk TG content varied from 20 to $110 \mathrm{mg} / \mathrm{ml}$, somewhat lower than that reported by Chalk \& Bailey (1979), Grigor \& Warren (1980) and Keen et al. (1981) (range from 120 to $180 \mathrm{mg} / \mathrm{ml}$ ), but was comparable to that reported by Chu \& Cox (1970). Previously, the concentration of milk fat has been shown to be decreased (Godbole et al. 1981), unchanged (Chalk \& Bailey, 1979; Keen et al. 1981), or increased (Harzer et al. 1983) at different stages of lactation. We observed in the present study an increase in milk TG content at day 8 and occasionally at day 15 of lactation (Fig. 1). The explanation for these differences is not clear, but evidence has shown that milk volume and lipid content may be affected by many physiological and psychological factors (Crawford et al. 1976; Chalk \& Bailey, 1979; Hall, 1979). In the present study we found that the total content of milk fats was not affected by different dietary fats, but was modulated by the parity number. The extent of changes in TG content was greater in milk obtained from the dams bred only once or twice than from those bred three or four times. This finding was different from that seen in humans who reproduce frequently with short recuperation periods and show no significant effect on the milk fat content (Prentice et al. 1981; Merchant \& Martorell, 1988). It is possible that the synthesis of milk TG in rats, unlike humans, is modulated by the frequency of breeding.

In the mammary gland, milk TG is synthesized using fatty acids originating from the blood stream or from de novo synthesis (Smith \& Abraham, 1975). Since the levels of SFA, MUFA and PUFA during each lactation cycle were constant (Fig. 3), there appeared to be no significant effect of the length of lactation on the incorporation of fatty acids into milk TG. However, increasing the parity number changed the levels of SFA, MUFA and PUFA. In the SFO-fed dams, increasing the parity number increased the levels of SFA, but reduced the levels of MUFA and PUFA ( $n-3$ and $n-6)$. Opposite effects were observed in the EPOfed dams. We have also examined the SFA composition in milk TG. In both dietary groups the levels of 18:0 were similar and remained constant throughout the study. The levels of $8: 0,10: 0,12: 0,14: 0$ and 16:0 in milk TG increased progressively in the SFO-fed dams, whereas the levels of 8:0 and 10:0 reduced progressively in the EPO-fed dams (values not shown). These changes suggest that increasing the parity number increased the de novo synthesis of TG in the SFO-fed dams, but reduced it in the EPO-fed dams. An increase in the levels of MUFA and PUFA in the EPO-fed dams reflects an increase in the contribution of milk fatty acids from the blood stream, which might originate from the diet. It is concluded that the de novo synthesis of milk TG was affected by the type of fat in the maternal diet and by the parity number, but not by the duration of lactation. Indeed, the effect of maternal diet on milk TG fatty acid composition has been previously reviewed (Lammi-Keefe \& Jensen, 1984).

Our second objective was to examine whether supplementing the maternal diet with two different $n-6$ fatty acid-rich vegetable oils (SFO and EPO) would affect the $n-6$ fatty acid composition in milk fat sampled from different lactation periods in different breeding cycles. Our results show that the levels of $18: 2 n-6$ were generally constant in milk TG and progressively increased in milk PL (except in the fourth breeding cycle). This reflected a steady or an increasing influx of 18:2n-6 into milk from either the adipose tissues or the diet. The levels of $18: 2 n-6$ metabolites $(18: 3 n-6,20: 3 n-6$ and $20: 4 n-6)$ in milk TG (Fig. 4) fell progressively as lactation proceeded, whereas in milk PL they remained constant or 


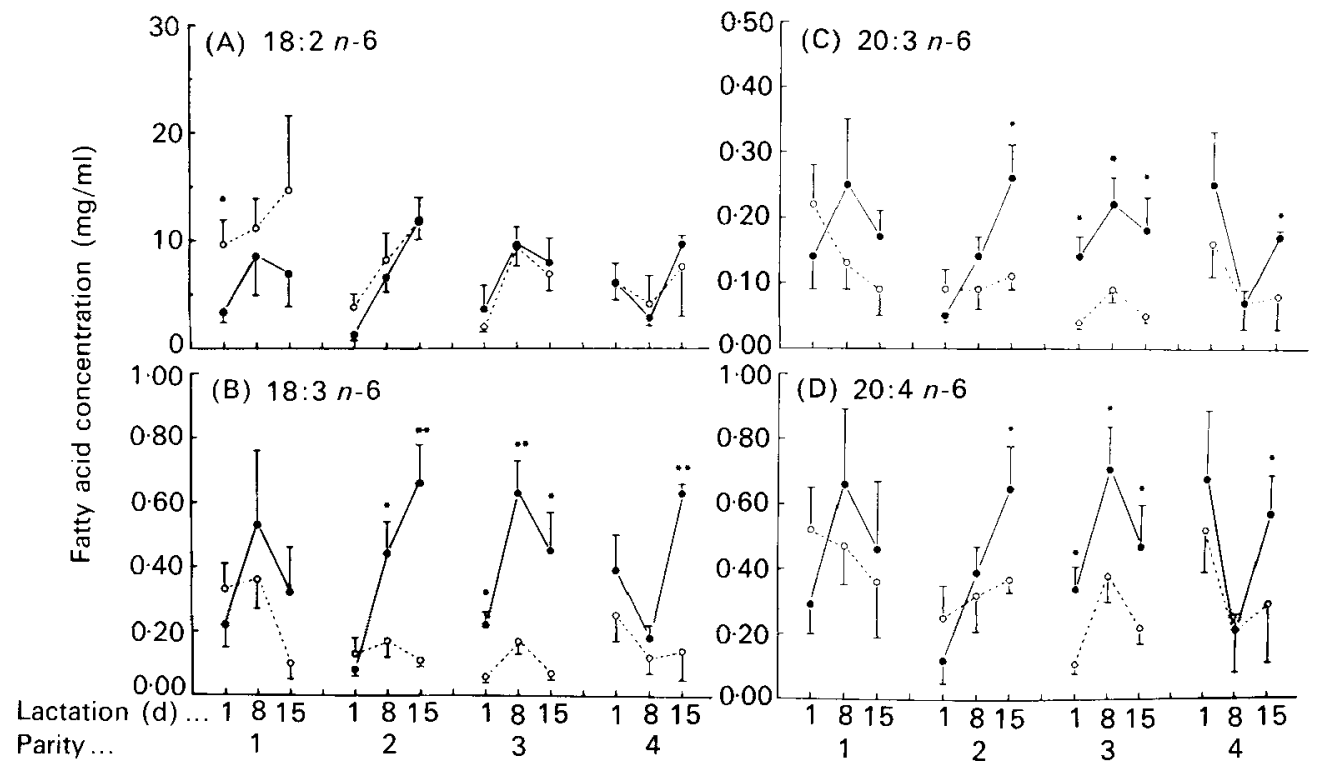

Fig. 6. Concentration $(\mathrm{mg} / \mathrm{ml})$ of (A) $18: 2 n-6$, (B) $18: 3 n-6$, (C) $20: 3 n-6$ and (D) $20: 4 n-6$ in rat milk triacylglycerols on days 1,8 and 15 of lactation. Milk samples were collected from the dams (values are presented in the order of once-, twice-, three- and four-times-bred animals) fed on a diet supplemented with either safflower oil $(\mathrm{SFO} ; \mathrm{O})$ or evening primrose oil $(\mathrm{EPO} ; O)$. Results are means with their standard errors, represented by vertical bars, for ten animals. Mean values for SFO were significantly different from those for EPO: *P<0.05, ${ }^{* *} P<0.01$. For details of diets, see Table $\mathrm{L}$.

were increased. Since TG represents $980 \mathrm{~g} / \mathrm{kg}$ milk fat, while PL represents only $10 \mathrm{~g} / \mathrm{kg}$, the overall levels of $18: 2 n-6$ metabolites were progressively reduced. This suggests that the incorporation of long-chain $n-6$ metabolites into milk TG, compared with their parent acid $18: 2 n-6$, was progressively reduced. Nevertheless, the levels of 6 -desaturated $(n-6)$ PUFA were consistently higher in milk TG or PL obtained from the EPO-fed than that from the SFO-fed dams. These differences were even more evident when the values were calculated in $\mathrm{mg} / \mathrm{ml}$ (Fig. 6) instead of $\mathrm{g} / 100 \mathrm{~g}$ total fatty acids, as shown in Fig. 5. The concentrations of $18: 3 n-6$ (Fig. 6(B)), 20:3n-6 (Fig. 6(C)) and 20:4n-6 (Fig. 6(D)), particularly after the second gestation-lactation cycle onwards, were consistently higher in the EPO group than in the SFO group, and more so at later lactation time (days 8 and 15). It is, thus, concluded that dietary $18: 3 n-6$, compared with $18: 2 n-6$, was more efficiently converted into $20: 3 n-6$ and $20: 4 n-6$ and that it and its metabolites were incorporated into milk TG. This conclusion is in agreement with the report of Hassam \& Crawford (1976) where they showed an increase in incorporation of $18: 3 n-6$ and its metabolites into milk collected from the $18: 3 n-6$ fed group.

The sum of $\mathrm{C}_{20}$ and $\mathrm{C}_{22} n-6$ fatty acids: the sum of $\mathrm{C}_{18} n-6$ fatty acids, $\left(\mathrm{C}_{20}+\mathrm{C}_{22}\right): \mathrm{C}_{18}$, has been previously used as an indicator for the conversion of $\mathrm{C}_{18} n-6$ to $\mathrm{C}_{20}$ and $\mathrm{C}_{22} n-6$ PUFA (Huang et al. 1991). Since the level of long-chain $\left(\mathrm{C}_{20}\right.$ and $\left.\mathrm{C}_{22}\right) n-6$ PUFA in milk collected from the SFO-fed dams decreased, whereas that of $18: 2 n-6$ either increased or did not change (Fig. $3(\mathrm{~A})$ ), a decrease in $\left(\mathrm{C}_{20}+\mathrm{C}_{22}\right):(18: 2 n-6+18: 3 n-6)$ resulted (Fig. 7). In other words, there may either be a decrease in the conversion of $\mathrm{C}_{18} n-6$ fatty acids into long-chain $n-6$ PUFA or a decrease in the incorporation of long-chain $n-6$ metabolites into milk fats during lactation. Similar changes were also observed in the EPO-fed dams. However, the values of long-chain $n$-6 PUFA were greater in the EPO than in the SFO group. Thus, the conversion of long-chain $n-6$ fatty acids was less active in the SFO group, 


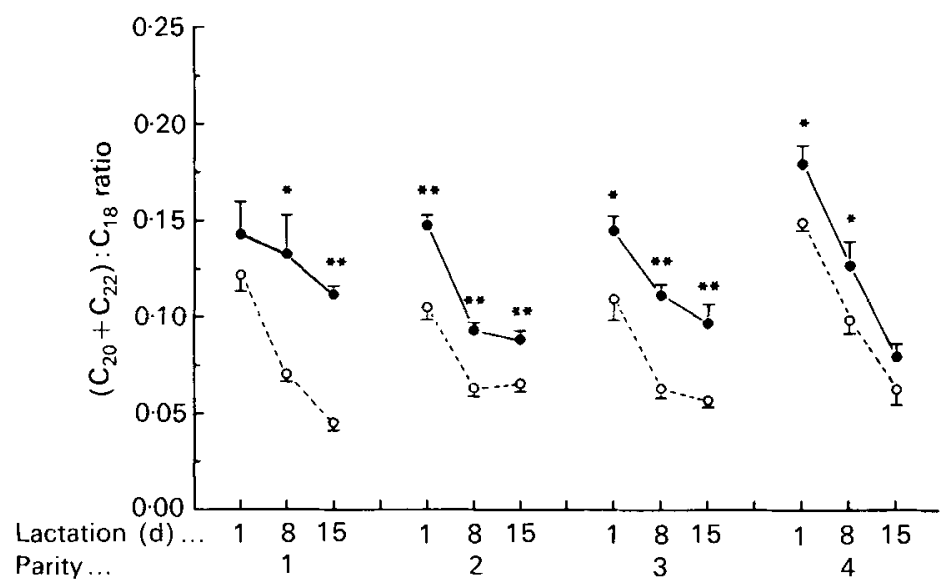

Fig. 7. Mean $\mathrm{C}_{20}+\mathrm{C}_{22} n-6: \mathrm{C}_{18} n-6$ ratio of rat milk triacylglycerol collected on days 1,8 , and 15 of lactation from the dams (values are presented in the order of once-, twice-, three- and four-times-bred animals) fed on a diet supplemented with either safflower oil (SFO;O) or evening primrose oil (EPO; $)$ ). Results are means with their standard errors, represented by vertical bars, for ten animals. Mean values for SFO were significantly different from those for EPO: ${ }^{*} P<0 \cdot 05,{ }^{* *} P<0 \cdot 01$. For details of diets, see Table 1.

possibly as a result of inhibition of $\Delta^{6}$ desaturation by high $18: 2 n-6$ levels (Brenner, 1981). On the other hand, the formation of long-chain $n-6$ PUFA was more effective in the EPOfed dams because dietary $18: 3 n-6$ bypassed such inhibition. This may also explain why milk from mothers fed on a high-PUFA diet (mainly $18: 2 n-6$ ), contains less $20: 3 n-6$ and $20: 4 n$ 6 (Ballabriga \& Martinez, 1976; Mellies et al. 1979).

These differences in long-chain $n-6$ PUFA content between the SFO and EPO groups may be of some nutritional significance. Long-chain $n-6$ PUFA required by the young may be supplied by the milk or may depend on the ability of pups to form them from $18: 2 n$ 6 absorbed from the milk. It has been questioned whether the neonate, particularly when born prematurely, is capable of synthesizing long-chain PUFA in sufficient amounts (Clandinin et al. 1981). In neonates with reduced desaturase activity (for example, as appears to be the case in human atopic disorders, Strannegard et al. 1987) a greater volume of milk must be consumed in order to meet the need for long-chain $n$-6 PUFA. Also, if the mother is deficient in $\Delta^{6}$-desaturase activity, as appears to occur in atopic eczema (Wright, 1990) and may occur in diabetes (Tilivis \& Miettinen, 1985), her milk may contain reduced amounts of long-chain EFA. Raising the levels of long-chain $n-6$ metabolites in milk by modulating the maternal diet may be a feasible approach to correcting such deficits, since the present study shows that the levels of long-chain $n-6$ in milk may be increased by supplementing the maternal diet with long-chain n-6 PUFA, e.g. 18:3n-6.

The authors are grateful to Dr Richard Cantrill for editorial assistance, and to Mr Rick Smith and Ms Xiaorong Lin for technical assistance.

\section{REFERENCES}

Ballabriga, A. \& Martinez, M. (1976). Changes in erythrocyte lipid stoma in the premature infant according to dietary fat composition. Acta Paediatrica Scandinavica 65, 705 709.

Brandorff, N. P. (1980). The effect of dietary fat on the fatty acid composition of lipid secreted in rat's milk. Lipids 15, 276-278.

Brenner, R. R. (1981). Nutritional and hormonal factors influencing desaturation of essential fatty acids. Progress in Lipid Research 20, 41-47.

Chalk, P. A. \& Bailey, E. (1979). Changes in the yield, and carbohydrate, lipid and protein content of milk during lactation in the rat. Journal of Developmental Physiology 1, $61-79$.

Chu, R. C. \& Cox, D. H. (1970). Excessive dietary zinc during lactation and nutritive value and mineral composition of rat's milk. Nutrition Reports International 2, 179-184. 
Clandinin, M. T., Chapell, J. E. \& Heim, T. (1981). Fatty acid accretion in fetal and neonatal liver: implications for fatty acid requirements. Early Human Development 5, 7-14.

Clandinin, M. T., Chappell, J. E., Leong, S., Heim, T., Swyer, P. R. \& Chance, G. W. (1980). Intrauterine fattyacid accretion rates in human brain: implications for fatty-acid requirements. Early Human Developmen 4 , $121-130$

Crawford, M. A., Hall, B., Laurance, B. M. \& Munhumbo, A. (1976). Milk lipids and their variability. Current Medical Research and Opinion 1 Suppl. 4, 3343.

Folch, J., Lees, M. \& Sloane-Stanley, G. H. (1957). A simple method for the isolation and purification of total lipids from animal tissues. Journal of Biological Chemistry 226, 497-509.

Godbole, V. Y., Grundleger, M. L., Pasquine, T. A. \& Thenen, S. W. (1981). Composition of rat milk from day 5 to 20 of lactation and milk intake of lean and preobese Zucker pups. Journal of Nutrition 111, $480-487$.

Grigor, M. R. \& Warren, S. M. (1980). Dietary regulation of lipogenesis in lactating rats. Biochemical Journal 188, $61-65$.

Grossman, S. H., Mollo, E. \& Ertingshausen, G. (1976). Simplified, totally enzymatic method for determination of serum triglycerides with a centrifugal analyzer. Clinical Chemistry 22, 1310-1313.

Hall, B. (1979). Uniformity of human milk. American Journal of Clinical Nutrition 32, 304-312.

Harzer, G., Haug, M., Dieterich, I. \& Gentner, P. R. (1983). Changing patterns of human milk lipids in the course of the lactation and during the day. American Jounal of Clinical Nutrition 37, 612-621.

Hassam, A. G. \& Crawford, M. A. (1976). Influence of maternal dietary $\gamma$-linolenic acid on the milk and liver lipids of suckling rats. Nutrition \& Metabolism 20, 112-116.

Huang, Y.-S., Smith, R. S., Redden, P. R., Cantrill, R. C. \& Horrobin, D. F. (1991). Modification of liver fatty acid metabolism in mice by $n-3$ and $n-6 \Delta^{6}$ desaturase substrates and products. Biochimica et Biophysica Acta $1082,319-327$.

Jenness, R. (1974). The composition of milk. In Lactation: A Comprehensive Treatise, vol. 3, pp. 3-107 [B. L. Larson and V. R. Smith, editors]. New York: Academic Press.

Jensen, R. D. (1989). The Lipids of Human Milk. Boca Raton, FL: CRC Press Inc.

Jensen, R. D., Hagerty, M. M. \& McMahon, K. E. (1978). Lipids of human milk and infant formulas : a review. American Journal of Clinical Nutrition 31, 990-1016.

Keen, C. L., Lönnerdal, B., Clegg. M. \& Hurley, L. S. (1981). Developmental changes in composition of rat milk: trace elements, minerals, protein, carbohydrate and fat. Journal of Nutrition 111, 226-230.

Lammi-Keefe, C. J. \& Jensen, R. G. (1984). Lipids in human milk: a review. 2. Composition and fat-soluble vitamins. Journal of Pediatric Gastroenterology and Nutrition 3, 172-198.

Linzell, J. L., Mepham, T. B., Annison, E. F. \& West, C. E. (1969). Mammary metabolism in lactating sows: arteriovenous differences of milk precursors and the mammary metabolism of $\left[{ }^{14} \mathrm{C}\right] \mathrm{glucose}$ and $\left[{ }^{14} \mathrm{C}\right]$ acetate. British Journal of Nutrition 23, 319-332.

McBurney, J. J., Meier, H. \& Hoag, W. G. (1964). Device for milking mice. Journal of Laboratory and Clinical Medicine 64, 485-487.

Mellies, M. J., Ishikawa, T. T., Gartside, P. S., Burton, K., MacGee, J., Allen, K., Steiner, P. M., Brady, D. \& Glueck, C. J. (1979). Effects of varying maternal dietary fatty acids in lactating women and their infants. American Journal of Clinical Nutrition 32, 299-303.

Merchant, K. \& Martorell, R. (1988). Frequent reproductive cycling: does it lead to nutritional depletion of mothers? Progress in Food and Nutrition Science 12, 339--369.

Mills, D. E., Ward, R. P. \& Huang, Y.-S. (1990). Fatty acid composition of milk from genetically normotensive and hypertensive rats. Journal of Nutrition 120, $431-435$.

Morrison, W. R. (1968). The distribution of phospholipids in some mammalian milks. Lipids 3, $101-103$.

Morrison, W. R. \& Smith, L. M. (1964). Preparation of fatty acid methyl esters and dimethylacetals from lipids with boron trifluoride methanol. Journal of Lipid Research 5, 600-608.

Prentice, A., Prentice, A. M. \& Whitehead, R. G. (1981). Breast-milk fat concentrations of rural African women. 2. Long-term variations within a community. British Journal of Nutrition 45, 495-503.

Smith, S. \& Abraham, S. (1975). The composition and biosynthesis of milk fat. Advances in Lipid Research 13, 195-239.

Snedecor, G. W. \& Cochran, W. G. (1967). Statistical Methods. Ames, Iowa: Iowa State University Press.

Spincer, J., Rook, J. A. F. \& Towers, K. G. (1969). The uptake of plasma constituents by the mammary gland of the sow. Biochemical Journal 111, 727--732.

Strannegard, I. L., Svennerholm, L. \& Strannegard, O. (1987). Essential fatty acids in serum lecithin of children with atopic dermatitis and in umbilical cord serum of infants with high or low IgE levels. International Archives of Allergy and Applied Immunology 82, 422-423.

Tilivis, R.S. \& Miettinen, T. A. (1985). Fatty acid composition of serum lipids, erythrocytes and platelets in insulin-dependent diabetic women. Journal of Clinical Endocrinology and Metabolism 61, 741-745.

Wright, S. (1990). Essential fatty acids and atopic eczema: biochemical and immunological studies. In Omega-6 Essential Fatty Acids: Pathophysiology and Roles in Clinical Medicine, pp. 55 65 [D. F. Horrobin, editor]. New York: Alan R. Liss Inc. 